OPEN ACCESS

Edited by:

Maria Concepcion Garcia Otaduy,

University of São Paulo, Brazil

Reviewed by:

Jeffrey A. Stanley,

Wayne State University, United States

Frank P. MacMaster,

University of Calgary, Canada

${ }^{*}$ Correspondence:

Pallab Bhattacharyya

bhattap@ccf.org

Specialty section

This article was submitted to Neuroimaging and Stimulation,

a section of the journal

Frontiers in Psychiatry

Received: 07 February 2021 Accepted: 08 November 2021 Published: 03 December 2021

Citation:

Bhattacharyya P, Anand A, Lin J and Altinay M (2021) Left Dorsolateral Prefrontal Cortex G/x/tCr Predicts Efficacy of High Frequency 4- to 6-Week rTMS Treatment and Is

Associated With Symptom Improvement in Adults With Major Depressive Disorder: Findings From a Pilot Study.

Front. Psychiatry 12:665347. doi: 10.3389/fpsyt.2021.665347

\section{Left Dorsolateral Prefrontal Cortex GIx/tCr Predicts Efficacy of High Frequency 4- to 6-Week rTMS Treatment and Is Associated With Symptom Improvement in Adults With Major Depressive Disorder: Findings From a Pilot Study}

\author{
Pallab Bhattacharyya ${ }^{1,2 \star}$, Amit Anand ${ }^{3}$, Jian Lin $^{1}$ and Murat Altinay ${ }^{3}$ \\ ${ }^{1}$ Cleveland Clinic, Imaging Institute, Cleveland, OH, United States, ${ }^{2}$ Department of Radiology, Cleveland Clinic Lerner \\ College of Medicine, Cleveland, $\mathrm{OH}$, United States, ${ }^{3}$ Cleveland Clinic, Neurological Institute, Cleveland, $\mathrm{OH}$, United States
}

About $20-40 \%$ of estimated 121 million patients with major depressive disorder (MDD) are not adequately responsive to medication treatment. Repetitive transcranial magnetic stimulation (rTMS), a non-invasive, non-convulsive neuromodulation/neurostimulation method, has gained popularity in treatment of MDD. Because of the high cost involved in rTMS therapy, ability to predict the therapy effectiveness is both clinically and cost wise significant. This study seeks an imaging biomarker to predict efficacy of rTMS treatment using a standard high frequency $10-\mathrm{Hz}$ 4- to 6-week protocol in adult population. Given the significance of excitatory and inhibitory neurotransmitters glutamate (Glu) and gamma aminobutyric acid (GABA) in the pathophysiology of MDD, and the involvement of the site of rTMS application, left dorsolateral prefrontal cortex (IDLPFC), in MDD, we explored IDLPFC GIx (Glu + glutamine) and GABA levels, measured by single voxel magnetic resonance spectroscopy (MRS) with total creatine (tCr; sum of creatine and phosphocreatine) as reference, as possible biomarkers of rTMS response prediction. Mescher-Garwood point-resolved spectroscopy (MEGA-PRESS) MRS data from 7 patients (40-74 y) were used in the study; 6 of these patients were scanned before and after 6 weeks of $r$ TMS therapy. Findings from this study show inverse correlation between pretreatment IDLPFC GIx/tCr and (i) posttreatment depression score and (ii) change in depression score, suggesting higher Glx/tCr as a predictor of treatment efficacy. In addition association was observed between changes in depression scores and changes in $\mathrm{Gl} \times \mathrm{t} \mathrm{tCr}$ ratio. The preliminary findings did not show any such association between GABA/tCr and depression score.

Keywords: repetitive transcranial magnetic stimulation (rTMS), major depressive disorder (MDD), magnetic resonance spectroscopy (MRS), glutamate, gamma aminobutyric acid (GABA) 


\section{INTRODUCTION}

Major depressive disorder (MDD), which has a lifetime prevalence of $15 \%$ (1), does not respond adequately to medication treatment in $\sim 20-40 \%$ of affected patients (2), and these patients have higher morbidity and mortality than those with disease that responds to medication $(3,4)$. Although electrical stimulation techniques such as electroconvulsive therapy (57), vagus nerve stimulation (8-10), and deep brain stimulation (11-13) are suitable for medication-resistant MDD, they are invasive in nature. Repetitive transcranial magnetic stimulation (rTMS), on the other hand, is a non-invasive, non-convulsive neuromodulation/neurostimulation method that has gained popularity for the treatment of MDD that is not responsive to medication (14-37). In particular, high-frequency $(>5 \mathrm{~Hz})$ rTMS applied to the left dorsolateral prefrontal cortex (IDLPFC) has been found to significantly decrease Hamilton Depression Rating Scale (HAM-D) scores in patients with medicationresistant MDD $(38,39)$. Standard and most optimal rTMS therapies are administered at a frequency of $10 \mathrm{~Hz}(40,41)$ over 4-6 weeks (40).

In patients with MDD, rTMS has been reported to change the balance of excitation and inhibition in cortical networks $(42,43)$, and the antidepressant effect from rTMS has been attributed in part on modulation of the major excitatory neurotransmitter glutamate (Glu) and the major inhibitory neurotransmitter gamma aminobutyric acid (GABA) (44). Multiple studies have documented the involvement of these neurotransmitters in the pathophysiology of MDD (45-51). Changes in cortical Glu or Glx (Glu + glutamine) and GABA levels in patients with MDD have been investigated using in vivo magnetic resonance spectroscopy (MRS). In spite of some differences in acquisition (e.g., Mescher-Garwood point-resolved spectroscopy [MEGA-PRESS] vs. short echo time [TE] PRESS), analysis, and quantification methodologies (e.g., absolute levels vs. ratios), these studies have demonstrated a reduction in cortical Glu or Glx and GABA levels associated with MDD (45, 48-50, 52). More specifically, reduced PFC Glx level in patients with MDD has been reported in several studies $(45,53,54)$. Researchers have suggested that dysfunction of the glutamatergic system and malfunction in Glu metabolism are contributing factors to the neurobiology and pathophysiology of $\operatorname{MDD}(55,56)$, and the efficacy of glutamatergic agents (glutamatergic targets/receptors such as ketamine, mamantine, riluzole, dextromethorphan, AZD6765 etc.) for the treatment of MDD has been reported $(56,57)$. Studies have also shown that reduced cortical GABA level is associated with dysfunctional GABAergic interneurons and $\mathrm{GABA}_{\mathrm{A}}$ receptors; affected GABAergic transmission has been proposed as a mechanism of MDD (58-60). rTMS studies have shown deficits in cortical inhibition in adults with MDD (61, 62); while in children and adolescents increased excitatory cortical facilitation with unchanged cortical inhibition was observed (63).

Multiple in vivo studies of Glu and GABA modulation after rTMS in patients with MDD have been performed (58, 6466). In one study using MEGA-PRESS, the medial prefrontal cortex (MPFC) Glu level was unchanged but the GABA level was elevated after 25 sessions of $10-\mathrm{Hz}$ rTMS therapy applied at the IDLPFC (58). In another study using PRESS and involving 10 sessions of $20-\mathrm{Hz}$ rTMS, an increase in Glu level was seen in the DLPFC, with no changes seen in the anterior cingulate cortex (64). In a short TE PRESS study of young adults treated with $10-\mathrm{Hz}$ rTMS for 15 days, the IDLPFC Glu level was increased in responders but reduced in non-responders (65). Another study using MEGA-PRESS demonstrated an increase in DLPFC GABA level after 6 weeks of $10-\mathrm{Hz}$ rTMS therapy (67).

The prefrontal cortex has been shown to be important in the pathogenesis of $\operatorname{MDD}(68,69)$, and decreased activation of the cortical areas of the mood-regulating circuit has also been reported in patients with MDD $(70,71)$. More specifically, several studies have shown abnormalities in the DLPFC in patients with MDD (72-76), with affected patients demonstrating reduced levels of GABA and Glx (Glu + glutamine [Gln]) in the DLPFC $(45,77,78)$. Lower metabolic activity in the DLPFC (79) as well as lower functional connectivity within the cognitive control network (80), a network that contains the DLPFC, has been reported in depression. In addition MDD is associated with reduced prefrontal cortex gray matter volume, cell counts and glucose metabolism (81). These abnormal (mostly left) prefrontal cortex activities in MDD therefore make the DLPFC a logical and popular rTMS target $(73,81-83)$.

Differences in Glu levels between responders and nonresponders to antidepressants (84) and rTMS therapy $(64,65)$ suggest that Glu level is a predictor of therapy outcomes in MDD. More specifically, studies have demonstrated that responders to rTMS therapy have lower baseline DLPFC Glu levels than non-responders $(64,65)$, suggesting that baseline Glu level could be a predictor of response to rTMS therapy. However, most of these studies included only young adults or were carried out over a different period of time than the standard and optimal 4- to 6-week period (40). Thus, additional research is needed to establish an imaging biomarker that can be used to predict the success of rTMS treatment using a standard $10-\mathrm{Hz}(40,41) 4$ - to 6 -week protocol in the adult population. Identifying such biomarker is significant from out of pocket patient expense also, since rTMS therapy is quite costly (can range from $\sim 6,000$ to $\sim \$ 15,000$ for 30 sessions in the USA depending on the location, center, applicable discounts and insurance coverage) and is often not covered by insurance.

In this longitudinal study, we measured $\mathrm{Glx} / \mathrm{tCr}$ and $\mathrm{GABA} / \mathrm{tCr}$ at the IDLPFC, the site of rTMS application, to determine whether the baseline measures of these could be used to predict outcomes after 6 weeks of $10-\mathrm{Hz}$ rTMS therapy. To this end, we assessed the association between these baseline ratios ( $\mathrm{Glx} / \mathrm{tCr}$ and $\mathrm{GABA} / \mathrm{tCr}$ ) and change in 17-item Hamilton Depression (HAM-D) score after rTMS, as well as the association between the baseline ratios and posttreatment HAM-D score. In addition, we evaluated the $\mathrm{Glx} / \mathrm{tCr}$ and $\mathrm{GABA} / \mathrm{tCr}$ ratios to track recovery after rTMS therapy, i.e., we assessed the association between the changes of these ratios and HAM-D scores in response to rTMS therapy. 


\section{MATERIALS AND METHODS}

The study was performed following an IRB-approved protocol. All patients provided written informed consent. We initially enrolled 12 patients ( 4 men; mean age, $53 \mathrm{y} \pm 15 \mathrm{y}$; range, $23-74$ y) who had an HAM-D score $>15$ and who met the DSM-IV-TR (85) criteria for MDD inadequately responsive to at least one antidepressant despite treatment with an adequate dosage for at least 8 weeks (the indication for rTMS approved by the Food and Drug Administration). Patients were recruited from Center of Behavioral Health outpatient psychiatry clinic for mood disorders at our center. Two of these patients did not complete the study, undergoing only $1 \mathrm{MR}$ imaging session and 3 patients had excessive motion during the pretreatment scan; thus, the final analysis consisted of 7 patients.

Of the 7 subjects included in the final analysis, 6 subjects were on antidepressants in combination with low dose 2nd generation neuroleptics $(n=4)$, mood stabilizers $(n=2)$, stimulants $(n=2)$ and other augmentation agents $(n=2)$. Low dose anti-anxiety medications were allowed per inclusion criteria $(n=4)$. Patients were asked to remain on the same dosages on all of the medications during the course of the rTMS treatment. No new medications and/or other non-medication treatment modalities were started at least 1 month before or during the acute rTMS series.

\section{rTMS Protocol}

rTMS was performed using a MagPro R-30 magnetic stimulator (MagVenture, Farum, Denmark) with "cool B-65" magnetic coil, a device that has been used effectively in previous studies $(14,86,87)$. Each patient underwent rTMS therapy sessions 5 times per week for a total of 6 weeks (total of 30 rTMS sessions); we selected a duration of 6 weeks because previous studies have used 4-6 weeks of treatment to testy for rTMS effectiveness $(14,88,89)$. Each session lasted $\sim 40 \mathrm{~min}$ and used the following parameters: frequency, $10 \mathrm{~Hz}$; power, $120 \%$ of the motor threshold (i.e., minimum amount of energy needed to trigger thumb movement); duration of stimulus, $4 \mathrm{~s}$; intertrain interval, $26 \mathrm{~s}$; number of pulses per train, 75; and total number of pulses, 3,000. In order to locate the IDLPFC, first the left motor strip controlling the movements of the right thumb was located. The coil was then advanced $5 \mathrm{~cm}$ on to the anterior of the motor strip to target the IDLPFC. An experienced staff psychiatrist (MA) administered the rTMS and also performed HAM-D assessment at baseline and every 2 weeks.

\section{MR Imaging}

MR scans were performed on a Siemens 3T Prisma scanner (Erlangen, Germany) using a 20-channel coil head/neck coil. Each patient was scanned within 1 week before starting rTMS therapy (pretreatment scan) and within 1 week after the end of 6 weeks of therapy (posttreatment scan).

Each MR session consisted of the following scans: (1) Localizer scan to obtain scout images: scan time, $9 \mathrm{~s}$; (2) Gradient recalled echo scan for field-mapping: 32 axial slices; thickness, $4 \mathrm{~mm}$; field of view (FOV), $256 \mathrm{~mm} \times 256 \mathrm{~mm}$; dual echo times (TE1/TE2)/repetition time (TR)/flip angle (FA), 4.89 $\mathrm{ms} / 7.35 \mathrm{~ms} / 388 \mathrm{~ms} / 60^{\circ}$; matrix, $64 \times 64$; bandwidth, $260 \mathrm{~Hz}$; scan time, $36 \mathrm{~s}$; (3) T1-weighted anatomical magnetization prepared rapid acquisition gradient echo (MPRAGE) scan: 120 axial slices; thickness, $1.2 \mathrm{~mm}$; FOV, $256 \mathrm{~mm} \times 256 \mathrm{~mm}$; inversion time/TE/TR/FA, $1,900 \mathrm{~ms} / 1.71 \mathrm{~ms} / 900 \mathrm{~ms} / 8^{\circ}$; matrix, $256 \times 128$; bandwidth, $62 \mathrm{kHz}$; scan time, $4 \mathrm{~min} 5 \mathrm{~s}$; and (4) Mescher-Garwood point-resolved spectroscopy (MEGAPRESS) scan for GABA and Glx measurement of a 2 $\times 2 \times 2 \mathrm{~cm}^{3}$ voxel in the IDLPFC: TR, $2,700 \mathrm{~ms}$; TE, $68 \mathrm{~ms}$; frequency-selective $180^{\circ}$ pulses at 1.9 (ON-resonance) and $1.5 \mathrm{ppm}$ (OFF-resonance, to minimize macromolecule contamination of GABA); minimum achievable frequency selective pulse bandwidth $(\sim 44 \mathrm{~Hz})$; number of averages, 128 per condition (ON-/OFF-resonance); weak water suppression (to use residual water fluctuation to assess patient motion); scan time, $10 \mathrm{~min} 53 \mathrm{~s}$. A trained technologist ensured that the IDLPFC voxel locations (Figure 1) were closely matched between the pretreatment and posttreatment sessions. The patients bit onto a bite-bar during all scans to reduce head motion. For all spectroscopy scans, shimming was performed using the FASTESTMAP shimming routine (90).

\section{MRS Data Analysis}

Postprocessing of MRS data was performed using the MRUI software package (91) following the method described by Bhattacharyya et al. (92). Postprocessing consisted of zero-order phase correction and frequency shift correction of the individual subspectra using residual water as a reference, averaging the individually phase- and frequency-corrected spectra, residual water suppression with Hankel-Lanczos squares singular value decomposition (HLSVD) filter (93), apodization by a $5-\mathrm{Hz}$ Gaussian filter, and zero filling The OFF-resonance spectrum was subtracted from the ON-resonance spectrum to obtain the final edited spectrum. Motion was identified retrospectively using residual water signal fluctuation as an indicator (92).

Next the $\sim 3.75$-ppm Glx and 3.01-ppm GABA peaks from the edited spectrum were fitted as double Gaussian peaks using the AMARES algorithm (94) with zero-order phase correction. The 3.04-ppm creatine ( $\mathrm{tCr}$ ) peak was fitted similarly from the OFF-resonance spectrum. $\mathrm{Glx} / \mathrm{tCr}$ and $\mathrm{GABA} / \mathrm{tCr}$ levels were obtained from $\mathrm{I}_{\mathrm{Gl}} / \mathrm{I}_{\mathrm{tCr}}$ and $\mathrm{I}_{\mathrm{GABA}} / \mathrm{I}_{\mathrm{tCr}}$, respectively, where $\mathrm{I}_{\mathrm{Glx}}$, $\mathrm{I}_{\mathrm{GABA}}$, and $\mathrm{I}_{\mathrm{tCr}}$ represent areas of the Glx, GABA, and $\mathrm{tCr}$ fits, respectively. Edited spectral fitting was done by including the $\sim 2.3 \mathrm{ppm}$ GABA+Glu and inverted NAA peaks as well, but that did not have any effect on $\mathrm{I}_{\mathrm{Glx}}$ or $\mathrm{I}_{\mathrm{GABA}}$.

\section{Statistical Analysis}

Percent (\%) changes in HAM-D score and $\mathrm{Glx} / \mathrm{Cr}$ were determined using the expressions

$$
\frac{(\text { Posttreatment HAMD })-(\text { Pretreatment HAMD })}{\text { Pretreatment HAMD }} \times 100
$$

and

$\frac{(\text { Posttreatment Glx/Cr })-(\text { Pretreatment Glx } / \mathrm{Cr})}{\text { Pretreatment Glx/Cr }} \times 100$ 

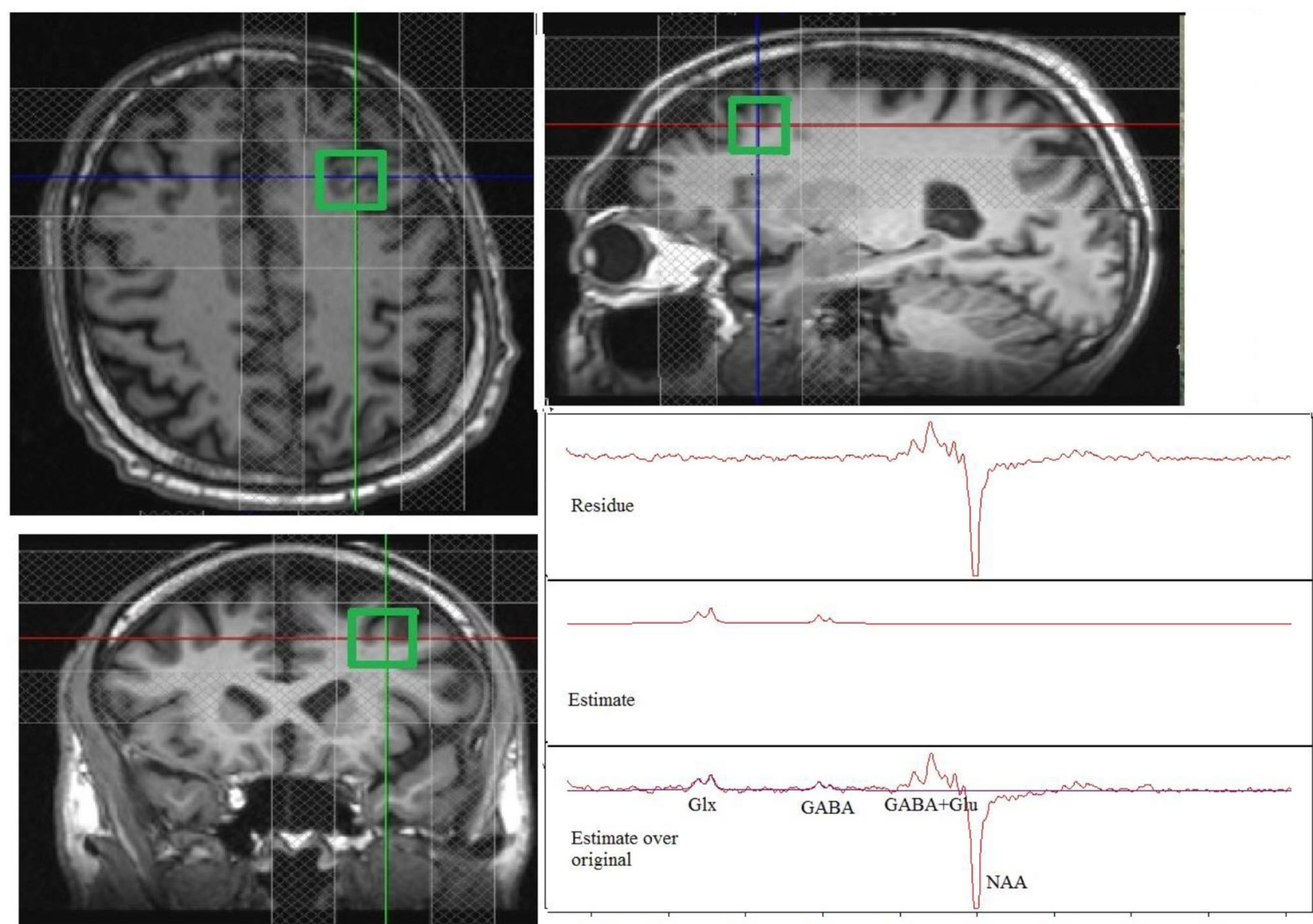

Estimate

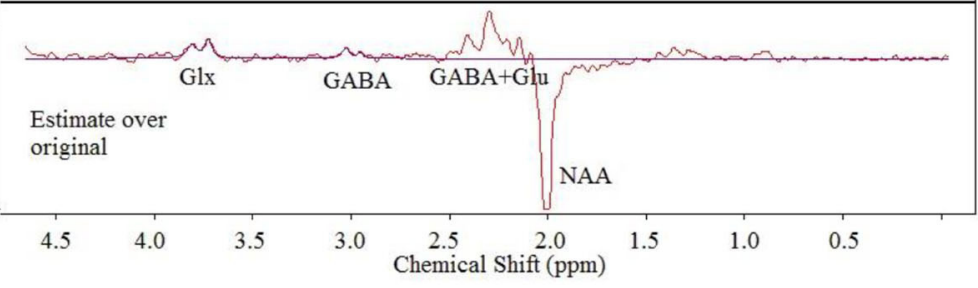

FIGURE 1 | Placement of a $2 \times 2 \times 2 \mathrm{~cm}^{3}$ dorsolateral prefrontal cortex voxel with the outer volume suppression bands and representative single-patient MEGA-PRESS edited spectra (original, estimate and residue). Glx, glutamate + glutamine; GABA, gamma aminobutyric acid; Glu, glutamate.

respectively. Non-parametric Wilcoxon signed rank test was between pre- and posttreatment HAM-D scores. Spearman correlation coefficient was used to characterize the association between (1) pretreatment DLPFC Glx/tCr (and GABA/tCr) ratios and changes in HAM-D scores (from pretreatment to 6 weeks posttreatment) and (2) changes in DLPFC Glx/tCr (and $\mathrm{GABA} / \mathrm{tCr}$ ) ratios and changes in HAM-D scores.

\section{RESULTS}

Some MRS datasets had to be discarded because of excessive motion. A total of 3 patients had pretreatment scans that could not be used because of excessive motion, and 1 of these patients also had a posttreatment scan that could not be used because of excessive motion. Thus, 7 motion-free pretreatment scans and 6 motion-free posttreatment scans were used for analysis. A Representative single-patient edited spectra (original, estimate and residual spectra) at the IDLPFC are shown in Figure 1.
From Wilcoxon signed rank test, significant decrease in HAMD scores was observed for the 10 patients who completed the study (pretreatment score, $20 \pm 3$; posttreatment score, $8 \pm 6$; $p=0.006$ ). Of the 7 patients (age: $59 \pm 13 \mathrm{y}$ ) with motion-free pretreatment scans the pretreatment and posttreatment HAM-D scores were $21 \pm 3$ and $11 \pm 8$, respectively ( $p=0.016$ ), while the pretreatment and posttreatment HAM-D scores for the 6 patients (age: $59 \pm 13 \mathrm{y}$ ) with both motion-free scans were $20 \pm 3$ and 10 \pm 8 , respectively $(p=0.031)$.

Overall, no significant changes in $\mathrm{Glx} / \mathrm{tCr}$ or $\mathrm{GABA} / \mathrm{tCr}$ were observed as a result of rTMS therapy (Table 1). Inverse Spearman correlations were observed between (1) posttreatment HAM-D score and pretreatment IDLPFC Glx/tCr $(n=7 ; p<0.0005)$ and (2) change in HAM-D score and pretreatment IDLPFC $\mathrm{Glx} / \mathrm{tCr}(n=7 ; p=0.001$; Figures 2A,B). No such significant correlations were observed between (1) posttreatment HAM-D score and pretreatment GABA/tCr $(n=7 ; p=0.66)$ and (2) change in HAM-D score and pretreatment IDLPFC GABA/tCr $(n=7 ; p=0.39)$. A significant correlation was observed between change in HAM-D score and change in Glx/tCr in the lDLPFC 
TABLE 1 | Depression ratings, GIx and GABA levels before and after rTMS treatment.

\begin{tabular}{|c|c|c|c|}
\hline Variable & Pretreatment value & Posttreatment value & $p$ \\
\hline HAM-D score $(n=10)$ & $20 \pm 3$ & $11 \pm 7$ & 0.0007 \\
\hline $\mathrm{G} \mid \mathrm{x} / \mathrm{t} \operatorname{Cr}(n=6)$ & $0.21 \pm 0.04$ & $0.24 \pm 0.05$ & 0.21 \\
\hline GABA/tCr $(n=6)$ & $0.11 \pm 0.02$ & $0.13 \pm 0.06$ & 0.20 \\
\hline
\end{tabular}

Data are presented as mean $\pm S D$. HAM-D, Hamilton Depression Rating Scale; Glx, glutamate + glutamine; tCr, total creatine; GABA, gamma aminobutyric acid.

( $n=6 ; p=0.02$; Figure 3); no such association was observed between change in HAM-D score and change in GABA/tCr $(n=6 ; p=0.45)$.

It should be pointed out that HAM-D scores were obtained every 2 weeks and similar analyses were run using the 4 -week HAM-D scores. Significant decrease in HAM-D scores were observed in 7 patients with motion-free pretreatment scans (4week HAM-D score $=10 \pm 7, p=0.022$ ). Similar to 6-week data, inverse Spearman correlations were seen between (1) 4week HAM-D score and pretreatment IDLPFC Glx/tCr $(n=7$; $p<0.0005)$ and (2) change in HAM-D score in 4 weeks and pretreatment IDLPFC Glx/tCr $(n=7 ; p<0.0005)$.

\section{DISCUSSION}

In this study, patients treated with 6 weeks of $10-\mathrm{Hz}$ rTMS targeting the IDLPFC demonstrated a decrease in HAM-D score; however, no overall changes in $\mathrm{Glx} / \mathrm{tCr}$ or $\mathrm{GABA} / \mathrm{tCr}$ ratios (averaged over 6 patients) were observed. One previous study reported no change in the MPFC Glu level after 25 sessions of $10-\mathrm{Hz}$ rTMS therapy (58); however, an increase in MPFC $\mathrm{GABA}+(\mathrm{GABA}+$ macromolecule) level was observed. Although this previous study had a higher number of patients $(n=23)$ than the current study, the region of interest (MPFC) was different from the site of rTMS application (IDLPFC), which was evaluated in the current study.

In this study, patients with higher pretreatment $\mathrm{Glx} / \mathrm{tCr}$ had lower posttreatment HAM-D scores and larger reductions in HAM-D score after 6 (as well as 4) weeks of rTMS. This finding, albeit from a small sample, is promising and suggests that IDLPFC Glu level may be a predictor of 4- to 6-week rTMS outcome. It should be noted that a higher baseline IDLPFC Glu level has also been previously reported in responders to antidepressant therapy (84), indicating that the predictive power of IDLPFC Glu level may not be limited to rTMS. On the other hand, a lower baseline Glu level has also been reported in youth responders to 3 weeks of rTMS (65), which is the opposite of what we observed in the current study. We speculate that this difference results from the difference in age groups between the studies. Cerebral Glu level has been reported to decrease with age (95); hence, in the older patient population as in this study (40-74 y for the patients who completed the study and had motion-free pretreatment scans), a higher pretreatment Glu level may favor the therapeutic action of rTMS. There was no overall change in Glx/tCr after rTMS. Our results indicate that while $\mathrm{Glx} / \mathrm{tCr}$ in the IDLPFC increased in
4 patients and decreased in 2 patients, a decrease in HAM$\mathrm{D}$ was associated with a lesser increase or larger decrease in $\mathrm{Glx} / \mathrm{tCr}$ ratio.

Baseline GABA level in this preliminary study was not associated with response to rTMS therapy, and no previous studies have demonstrated evidence of such a relationship. Additionally, no association between baseline prefrontal cortex GABA level and improvement in MDD was observed in a study assessing ketamine infusion therapy (96). It is likely, therefore, that the baseline GABA level does not predict recovery from MDD irrespective of the treatment regimen.

Test-retest reliability of Glx/Cr and GABA/Cr measurements of a $2 \times 2 \times 2 \mathrm{~cm}^{3}$ voxel in the IDLPFC using MEGA-PRESS sequence was evaluated independently in our center as described in the Supplementary Material. The test-retest variability (9.2\%) of $\mathrm{Glx} / \mathrm{Cr}$ is less than that observed in response to rTMS treatment, while the corresponding GABA/Cr changes for two subjects were less than the variability (16.6\%).

While a direct connection between Glx and excitatory neurotransmission is not obvious, it should be noted that Glx measured with the MEGA-PRESS sequence $(97,98)$ used in this study has been reported to contain mostly Glu with little or no Gln and is therefore considered a good measure of Glu $(58,99-$ 101). Based upon those reports, we speculate that much of our findings pertain to the involvement of excitatory Glu in rTMS therapy. However, we do recognize that there could be a small contribution of Gln in the Glx peak.

A higher IDLPFC $\mathrm{Cr}$ in MDD than in healthy controls has been reported (102). In this study, Glx/tCr and GABA/tCr ratios are reported, with areas of the respective resonances in the MEGA-PRESS edited spectra normalized to $\mathrm{tCr}$ area from OFFresonance spectra. For technical reasons, water-unsuppressed MEGA-PRESS scans were not incorporated in the protocol at the beginning of the study; however, those scans were added after the scans of the first 2 patients were completed. Normalizing Glx and GABA to $\mathrm{tCr}$ is a well-established method (103-105), and we validated this in our dataset by correlating Glx and GABA normalized to unsuppressed water with $\mathrm{Glx} / \mathrm{tCr}$ and GABA/tCr from all studies with unsuppressed water acquisition (i.e., from both MRI sessions for patients who completed the study and from pretreatment visits for patients who dropped out after $1 \mathrm{MRI}$ session). The 2 metrics were correlated ( $p=0.001$ for Glx and 0.0001 for GABA), which validated usage of ratio with respect to $t C r$ for this patient population.

Lack of any observed association of GABA in this preliminary study should be treated with caution. It is possible that the main 


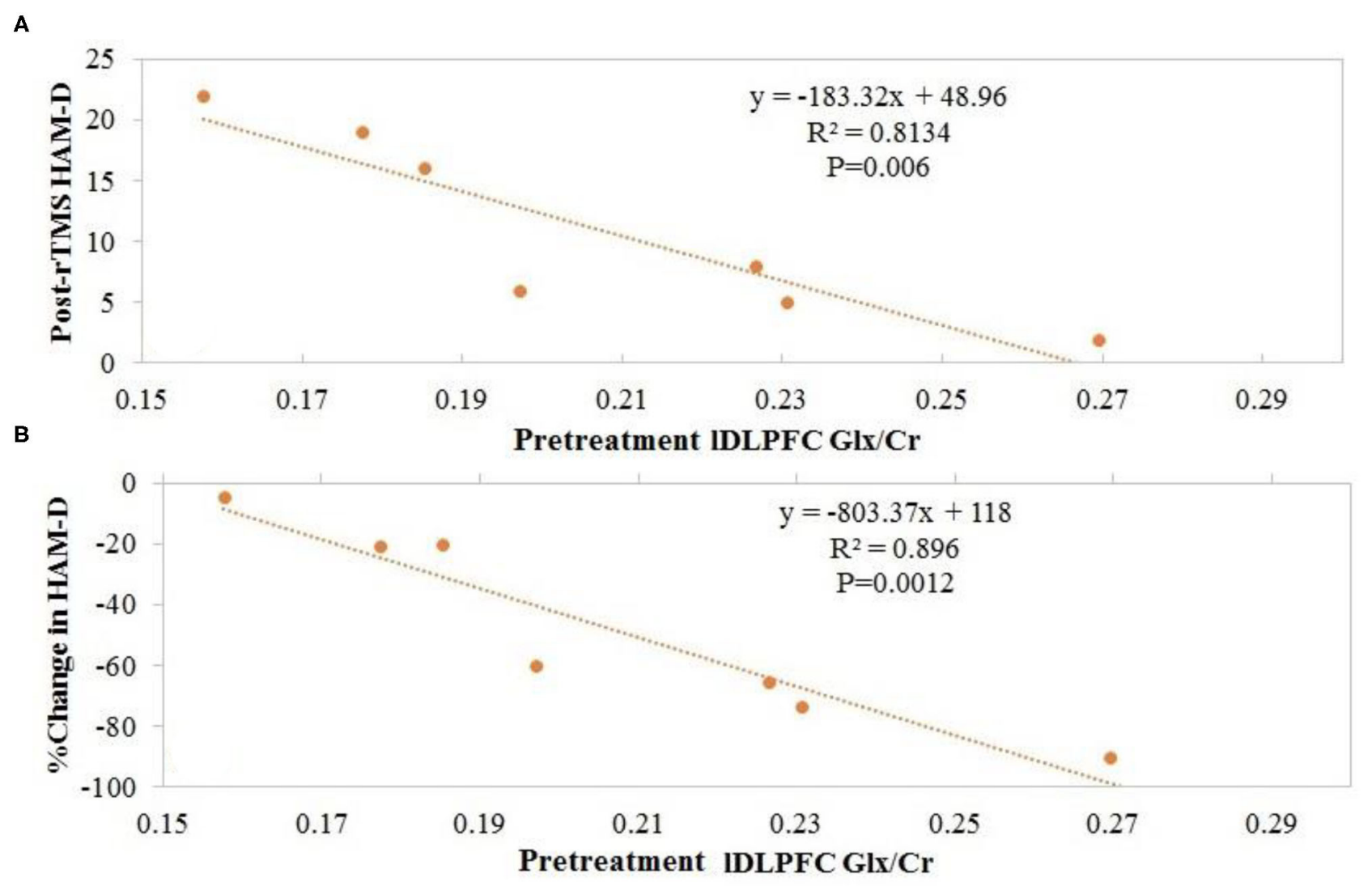

FIGURE 2 | Patients with higher pretreatment glutamate + glutamine (Glx)/total creatine (tCr) at the left dorsolateral prefrontal cortex (IDLPFC) demonstrated (A) lower posttreatment Hamilton Depression Rating Scale (HAM-D) scores and (B) greater change in HAM-D scores after repetitive transcranial magnetic stimulation (rTMS).

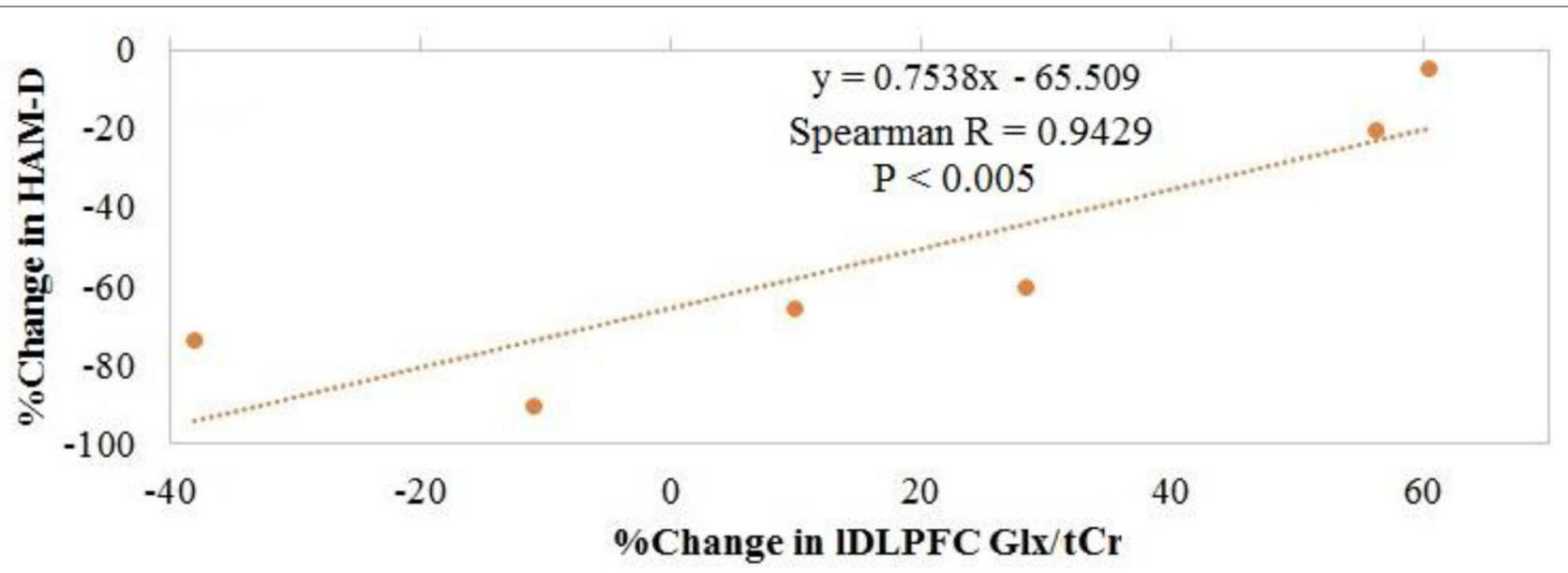

FIGURE 3 | Association between change in glutamate + glutamine (Glx)/total creatine (tCr) in the left dorsolateral prefrontal cortex and change in Hamilton Depression Rating Scale (HAM-D) score.

reason for the lack of any significant changes in $\mathrm{GABA} / \mathrm{tCr}$ ratios or any correlations therewith in this study is the lack of statistical power with 6 subjects. Spectral fitting error was $\sim 30 \%$ worse in GABA than in case of Glx, which would result in lower sensitivity of detecting GABA association. Signal to noise ratio and fitting error can be improved with GABA+ acquisition (103), but our choice of macromolecule-minimized GABA accounts for any inter-subject macromolecule level differences (106). 
This study had some limitations, including its small sample size. However, a power analysis with $n=7$ yielded power of 0.95 and 0.85 , respectively, for the inverse correlation observed between pretreatment IDLPFC Glx/tCr and change in HAM$\mathrm{D}$ and posttreatment HAM-D, respectively. In addition, with $n=6$ the study had power of 0.80 to detect $18 \%$ change in $\mathrm{Glx} / \mathrm{tCr}$ ratio. The study also did not include a sham treatment population, which may raise questions regarding glutamatergic involvement in the improvement of MDD as an effect of rTMS. Use of the standard 5-cm rule for rTMS target selection is another limitation, as use of neuronavigation instead has been shown to ensure more reliable, precise, and consistent targeting of the desired brain region (107). Finally, low doses of neuroleptics, benzodiazepine (not more than 1-2 mg), and mood stabilizers were allowed in the study; we did not assess the potential effects of these medications on the study findings. However, for all medications a fixed dose for 4 weeks (6 weeks for benzodiazepines) before rTMS with no change in medication during rTMS treatment was followed as part of the study protocol to minimize any medication effect to the observations reported in this study. We have covered a wide range of age in this study. We hypothesize that while the baseline metabolite levels may be varying due to age and drug regimen, the change in those levels in 6 weeks (study period) will be due to rTMS therapy.

\section{CONCLUSION}

This study found that the most commonly used rTMS protocol (10 Hz, 4-6 weeks, IDLPFC target) did not significantly change IDLPFC Glx/tCr or GABA/tCr ratios in adults with MDD. Patients with higher pretreatment IDLPFC Glx/tCr ratio did respond better to rTMS therapy; they had a greater reduction in HAM-D score and a lower posttreatment HAM-D score. These findings suggest that excitatory Glu is associated with recovery from MDD and can potentially be used as a biomarker to predict response to rTMS treatment, whereas no such relationship between inhibitory GABA and MDD/rTMS outcome was observed in this preliminary study. The results of this pilot study should be interpreted with caution because of the small sample size and absence of a sham arm; further studies using larger sample sizes are needed to assess these preliminary results.

\section{REFERENCES}

1. Tundo A, de Filippis R, Proietti L. Pharmacologic approaches to treatment resistant depression: evidences and personal experience. World J Psychiatry. (2015) 5:330-41. doi: 10.5498/wjp.v5.i3.330

2. Fava M. Diagnosis and definition of treatment-resistant depression. Biol Psychiatry. (2003) 53:649-59. doi: 10.1016/S0006-3223(03)00231-2

3. Russell JM, Hawkins K, Ozminkowski RJ, Orsini L, Crown WH, Kennedy $\mathrm{S}$, et al. The cost consequences of treatment-resistant depression. J Clin Psychiatry. (2004) 65:341-7. doi: 10.4088/JCP.v65n0309

4. Reutfors J, Andersson TM, Brenner P, Brandt L, DiBernardo A, Li G, et al. Mortality in treatment-resistant unipolar depression: a register-based cohort study in Sweden. J Affect Disord. (2018) 238:6749. doi: 10.1016/j.jad.2018.06.030

\section{DATA AVAILABILITY STATEMENT}

The raw data supporting the conclusions of this article will be made available by the authors, without undue reservation.

\section{ETHICS STATEMENT}

The studies involving human participants were reviewed and approved by Institutional Review Board, Cleveland Clinic. The patients/participants provided their written informed consent to participate in this study.

\section{AUTHOR CONTRIBUTIONS}

PB: MRI study design, planning, funding acquisition, MRI data analysis, and writing-original draft. AA: conceptualization, study design, writing-review, and editing. JL: MRI data processing. MA: patient recruitment and consenting, prescribing and administering rTMS, depression rating, writing-review, and editing. All authors contributed to the article and approved the submitted version.

\section{FUNDING}

Cleveland Clinic Research Program Committee partially funded this project.

\section{ACKNOWLEDGMENTS}

We are grateful to Sineyob Ahn from Siemens Healthineers for support with the MEGA-PRESS sequence used in this study. FASTESTMAP sequence, used for shimming, was developed by Edward J. Auerbach and Malgorzata Marjanska and were provided by the University of Minnesota under a C2P agreement. We also acknowledge Jennifer Bullen of Quantitative Health Sciences of Cleveland Clinic for her input in statistical analyses.

\section{SUPPLEMENTARY MATERIAL}

The Supplementary Material for this article can be found online at: https://www.frontiersin.org/articles/10.3389/fpsyt. 2021.665347/full\#supplementary-material

5. Kellner C. Review: maintenance antidepressants reduce risk of relapse in the 6 months following ECT in people with major depression. Evid Based Ment Health. (2014) 17:8. doi: 10.1136/eb-2013101663

6. de Vreede IM, Burger H, van Vliet IM. Prediction of response to ECT with routinely collected data in major depression. J Affect Disord. (2005) 86:323-7. doi: 10.1016/j.jad.2005.03.008

7. Ruedrich SL, Chu CC, Moore SL. ECT for major depression in a patient with acute brain trauma. Am J Psychiatry. (1983) 140:9289. doi: 10.1176/ajp.140.7.928

8. Muller $\mathrm{HH}$, Kornhuber J, Maler JM, Sperling W. The effects of stimulation parameters on clinical outcomes in patients with vagus nerve stimulation implants with major depression. J Ect. (2013) 29:e402. doi: 10.1097/YCT.0b013e318290f7ed 
9. Conway CR, Chibnall JT, Gangwani S, Mintun MA, Price JL, Hershey T, et al. Pretreatment cerebral metabolic activity correlates with antidepressant efficacy of vagus nerve stimulation in treatment-resistant major depression: a potential marker for response? J Affect Disord. (2012) 139:28390. doi: 10.1016/j.jad.2012.02.007

10. Cristancho P, Cristancho MA, Baltuch GH, Thase ME, O'Reardon JP. Effectiveness and safety of vagus nerve stimulation for severe treatment-resistant major depression in clinical practice after FDA approval: outcomes at 1 year. J Clin Psychiatry. (2011) 72:137682. doi: 10.4088/JCP.09m05888blu

11. Artigas F. Deep brain stimulation in major depression: plastic changes of 5-hydroxytryptamine neurons. Biol Psychiatry. (2014) 76:174-5. doi: 10.1016/j.biopsych.2014.05.008

12. Schlaepfer TE, Bewernick BH, Kayser S, Hurlemann R, Coenen VA. Deep brain stimulation of the human reward system for major depressionrationale, outcomes and outlook. Neuropsychopharmacology. (2014) 39:1303-14. doi: 10.1038/npp.2014.28

13. Schlaepfer TE, Bewernick BH. Deep brain stimulation for major depression. Handb Clin Neurol. (2013) 116:23543. doi: 10.1016/B978-0-444-53497-2.00018-8

14. Bakker N, Shahab S, Giacobbe P, Blumberger DM, Daskalakis ZJ, Kennedy SH, et al. rTMS of the dorsomedial prefrontal cortex for major depression: safety, tolerability, effectiveness, and outcome predictors for $10 \mathrm{~Hz}$ versus intermittent theta-burst stimulation. Brain Stimul. (2015) 8:208-15. doi: 10.1016/j.brs.2014.11.002

15. De Raedt R, Vanderhasselt MA, Baeken C. Neurostimulation as an intervention for treatment resistant depression: from research on mechanisms towards targeted neurocognitive strategies. Clin Psychol Rev. (2015) 41:61-9. doi: 10.1016/j.cpr.2014.10.006

16. McGirr A, Van den Eynde F, Tovar-Perdomo S, Fleck MP, Berlim MT. Effectiveness and acceptability of accelerated repetitive transcranial magnetic stimulation (rTMS) for treatment-resistant major depressive disorder: an open label trial. J Affect Disord. (2015) 173:216-20. doi: 10.1016/j.jad.2014.10.068

17. Tortella G, Selingardi PM, Moreno ML, Veronezi BP, Brunoni AR. Does noninvasive brain stimulation improve cognition in major depressive disorder? A systematic review. CNS Neurol Disord Drug Targets. (2014) 13:175969. doi: 10.2174/1871527313666141130224431

18. Chung SW, Hoy KE, Fitzgerald PB. Theta-burst stimulation: a new form of tms treatment for depression? Depress Anxiety. (2015) 32:18292. doi: 10.1002/da.22335

19. Boldt I, Eriks-Hoogland I, Brinkhof MW, de Bie R, Joggi D, von Elm E. Non-pharmacological interventions for chronic pain in people with spinal cord injury. Cochrane Database Syst Rev. (2014) 11:CD009177. doi: 10.1002/14651858.CD009177.pub2

20. Gaynes BN, Lloyd SW, Lux L, Gartlehner G, Hansen RA, Brode S, et al. Repetitive transcranial magnetic stimulation for treatment-resistant depression: a systematic review and meta-analysis. J Clin Psychiatry. (2014) 75:477-89; quiz 89. doi: 10.4088/JCP.13r08815

21. Leong $\mathrm{K}$, Chan $\mathrm{P}$, Grabovac A, Wilkins-Ho M, Perri $M$. Changes in mindfulness following repetitive transcranial magnetic stimulation for mood disorders. Can J Psychiatry. (2013) 58:687-91. doi: 10.1177/070674371305801206

22. Nakamura M. Therapeutic application of repetitive transcranial magnetic stimulation for major depression. Seishin Shinkeigaku Zasshi. (2012) 114:1231-49.

23. Lefaucheur JP, Andre-Obadia N, Poulet E, Devanne H, Haffen E, Londero A, et al. French guidelines on the use of repetitive transcranial magnetic stimulation (rTMS): safety and therapeutic indications. Neurophysiol Clin. (2011) 41:221-95. doi: 10.1016/j.neucli.2011. 10.062

24. Foucher JR, Luck D, Chassagnon S, Offerlin-Meyer I, Pham BT. What is needed for rTMS to become a treatment?. Encephale. (2007) 33:9829. doi: 10.1016/j.encep.2007.06.002

25. Fregni F, Marcolin MA, Myczkowski M, Amiaz R, Hasey G, Rumi DO, et al. Predictors of antidepressant response in clinical trials of transcranial magnetic stimulation. Int J Neuropsychopharmacol. (2006) 9:641-54. doi: 10.1017/S1461145705006280
26. Lam RW, Chan P, Wilkins-Ho $M$, Yatham LN. Repetitive transcranial magnetic stimulation for treatment-resistant depression: a systematic review and metaanalysis. Can J Psychiatry. (2008) 53:621-31. doi: 10.1177/070674370805300909

27. Pallanti S, Bernardi S. Neurobiology of repeated transcranial magnetic stimulation in the treatment of anxiety: a critical review. Int Clin Psychopharmacol. (2009) 24:163-73. doi: 10.1097/YIC.0b013e32832c2639

28. Richieri R, Adida M, Dumas R, Fakra E, Azorin JM, Pringuey $\mathrm{D}$, et al. Affective disorders and repetitive transcranial magnetic stimulation: therapeutic innovations. Encephale. (2010) 36(Suppl. 6):S197-201. doi: 10.1016/S0013-7006(10)70057-9

29. Schutter DJ, van Honk J. A framework for targeting alternative brain regions with repetitive transcranial magnetic stimulation in the treatment of depression. J Psychiatry Neurosci. (2005) 30:91-7.

30. Fitzgerald P. Repetitive transcranial magnetic stimulation and electroconvulsive therapy: complementary or competitive therapeutic options in depression? Australas Psychiatry. (2004) 12:234-8. doi: 10.1080/j.1039-8562.2004.02113.x

31. Padberg F, Moller HJ. Repetitive transcranial magnetic stimulation: does it have potential in the treatment of depression? CNS Drugs. (2003) 17:383403. doi: 10.2165/00023210-200317060-00002

32. Burt T, Lisanby SH, Sackeim HA. Neuropsychiatric applications of transcranial magnetic stimulation: a meta analysis. Int $J$ Neuropsychopharmacol. (2002) 5:73-103. doi: 10.1017/S1461145702002791

33. McNamara B, Ray JL, Arthurs OJ, Boniface S. Transcranial magnetic stimulation for depression and other psychiatric disorders. Psychol Med. (2001) 31:1141-6. doi: 10.1017/S0033291701004378

34. Boutros NN, Berman RM, Hoffman R, Miano AP, Campbell $\mathrm{D}$, Ilmoniemi R. Electroencephalogram and repetitive transcranial magnetic stimulation. Depress Anxiety. (2000) 12:166-9. doi: 10.1002/1520-6394(2000)12:3<166::AID-DA8>3.0.CO;2-M

35. Post RM, Kimbrell TA, McCann UD, Dunn RT, Osuch EA, Speer AM, et al. Repetitive transcranial magnetic stimulation as a neuropsychiatric tool: present status and future potential. J Ect. (1999) 15:39-59. doi: 10.1097/00124509-199903000-00005

36. O’Reardon JP, Solvason HB, Janicak PG, Sampson S, Isenberg KE, Nahas Z, et al. Efficacy and safety of transcranial magnetic stimulation in the acute treatment of major depression: a multisite randomized controlled trial. Biol Psychiatry. (2007) 62:1208-16. doi: 10.1016/j.biopsych.2007.01.018

37. Slotema CW, Blom JD, Hoek HW, Sommer IE. Should we expand the toolbox of psychiatric treatment methods to include Repetitive Transcranial Magnetic Stimulation (rTMS)? A meta-analysis of the efficacy of rTMS in psychiatric disorders. J Clin Psychiatry. (2010) 71:87384. doi: 10.4088/JCP.08m04872gre

38. Brunelin J, Poulet E, Boeuve C, Zeroug-vial H, d'Amato T, Saoud M. Efficacy of repetitive transcranial magnetic stimulation (rTMS) in major depression: a review. Encephale. (2007) 33:126-34. doi: 10.1016/S0013-7006(07)91542-0

39. Rizvi S, Khan AM. Use of transcranial magnetic stimulation for depression. Cureus. (2019) 11:e4736. doi: 10.7759/cureus.4736

40. McClintock SM, Reti IM, Carpenter LL, McDonald WM, Dubin M, Taylor SF, et al. Consensus recommendations for the clinical application of repetitive transcranial magnetic stimulation (rTMS) in the treatment of depression. $J$ Clin Psychiatry. (2018) 79:16cs10905. doi: 10.4088/JCP.16cs10905

41. Philip NS, Carpenter SL, Ridout SJ, Sanchez G, Albright SE, Tyrka $\mathrm{AR}$, et al. $5 \mathrm{~Hz}$ Repetitive transcranial magnetic stimulation to left prefrontal cortex for major depression. J Affect Disord. (2015) 186:137. doi: 10.1016/j.jad.2014.12.024

42. Fitzgerald PB, Fountain S, Daskalakis ZJ. A comprehensive review of the effects of rTMS on motor cortical excitability and inhibition. Clin Neurophysiol. (2006) 117:2584-96. doi: 10.1016/j.clinph.2006.06.712

43. Lenz M, Vlachos A. Releasing the cortical brake by noninvasive electromagnetic stimulation? rTMS induces LTD of GABAergic neurotransmission. Front Neural Circuits. (2016) 10:96. doi: 10.3389/fncir.2016.00096

44. Spronk D, Arns M, Fitzgerald PB. Repetitive transcranial magnetic stimulation in depression: protocols, mechanisms and new developments. In: Cohen, Evans E, editors. Neuromodulation and Neurofeedback: Techniques and Applications (2010). 
45. Hasler G, van der Veen JW, Tumonis T, Meyers N, Shen J, Drevets WC. Reduced prefrontal glutamate/glutamine and gammaaminobutyric acid levels in major depression determined using proton magnetic resonance spectroscopy. Arch Gen Psychiatry. (2007) 64:193-200. doi: 10.1001/archpsyc.64.2.193

46. Hashimoto K. Emerging role of glutamate in the pathophysiology of major depressive disorder. Brain Res Rev. (2009) 61:10523. doi: 10.1016/j.brainresrev.2009.05.005

47. Bernard R, Kerman IA, Thompson RC, Jones EG, Bunney WE, Barchas JD, et al. Altered expression of glutamate signaling, growth factor, and glia genes in the locus coeruleus of patients with major depression. Mol Psychiatry. (2011) 16:634-46. doi: 10.1038/mp.2010.44

48. Rosenberg DR, Macmaster FP, Mirza Y, Smith JM, Easter PC, Banerjee SP, et al. Reduced anterior cingulate glutamate in pediatric major depression: a magnetic resonance spectroscopy study. Biol Psychiatry. (2005) 58:7004. doi: 10.1016/j.biopsych.2005.05.007

49. Auer DP, Putz B, Kraft E, Lipinski B, Schill J, Holsboer F. Reduced glutamate in the anterior cingulate cortex in depression: an in vivo proton magnetic resonance spectroscopy study. Biol Psychiatry. (2000) 47:30513. doi: 10.1016/S0006-3223(99)00159-6

50. Chang L, Cloak CC, Ernst T. Magnetic resonance spectroscopy studies of GABA in neuropsychiatric disorders. J Clin Psychiatry. (2003) 64(Suppl. 3):7-14.

51. Lener MS, Niciu MJ, Ballard ED, Park M, Park LT, Nugent AC, et al. Glutamate and gamma-aminobutyric acid systems in the pathophysiology of major depression and antidepressant response to ketamine. Biol Psychiatry. (2017) 81:886-97. doi: 10.1016/j.biopsych.2016.05.005

52. Rosenberg DR, Mirza Y, Russell A, Tang J, Smith JM, Banerjee SP, et al. Reduced anterior cingulate glutamatergic concentrations in childhood OCD and major depression versus healthy controls. J Am Acad Child Adolesc Psychiatry. (2004) 43:1146-53. doi: 10.1097/01.chi.0000132812.44 $664.2 \mathrm{~d}$

53. Arnone D, Mumuni AN, Jauhar S, Condon B, Cavanagh J. Indirect evidence of selective glial involvement in glutamate-based mechanisms of mood regulation in depression: meta-analysis of absolute prefrontal neurometabolic concentrations. Eur Neuropsychopharmacol. (2015) 25:110917. doi: 10.1016/j.euroneuro.2015.04.016

54. Yuksel C, Ongur D. Magnetic resonance spectroscopy studies of glutamaterelated abnormalities in mood disorders. Biol Psychiatry. (2010) 68:78594. doi: 10.1016/j.biopsych.2010.06.016

55. Sanacora G, Treccani G, Popoli M. Towards a glutamate hypothesis of depression: an emerging frontier of neuropsychopharmacology for mood disorders. Neuropharmacology. (2012) 62:6377. doi: 10.1016/j.neuropharm.2011.07.036

56. Mathews DC, Henter ID, Zarate CA. Targeting the glutamatergic system to treat major depressive disorder: rationale and progress to date. Drugs. (2012) 72:1313-33. doi: 10.2165/11633130-000000000-00000

57. Jaso BA, Niciu MJ, Iadarola ND, Lally N, Richards EM, Park M, et al. Therapeutic modulation of glutamate receptors in major depressive disorder. Curr Neuropharmacol. (2017) 15:57-70. doi: 10.2174/1570159X14666160321123221

58. Dubin MJ, Mao X, Banerjee S, Goodman Z, Lapidus KA, Kang $\mathrm{G}$, et al. Elevated prefrontal cortex GABA in patients with major depressive disorder after TMS treatment measured with proton magnetic resonance spectroscopy. J Psychiatry Neurosci. (2016) 41:E37-45. doi: 10.1503/jpn.150223

59. Fogaca MV, Duman RS. Cortical GABAergic dysfunction in stress and depression: new insights for therapeutic interventions. Front Cell Neurosci. (2019) 13:87. doi: 10.3389/fncel.2019.00087

60. Maciag D, Hughes J, O’Dwyer G, Pride Y, Stockmeier CA, Sanacora G, et al. Reduced density of calbindin immunoreactive GABAergic neurons in the occipital cortex in major depression: relevance to neuroimaging studies. Biol Psychiatry. (2010) 67:465-70. doi: 10.1016/j.biopsych.2009.10.027

61. Bajbouj M, Lisanby SH, Lang UE, Danker-Hopfe H, Heuser I, Neu P. Evidence for impaired cortical inhibition in patients with unipolar major depression. Biol Psychiatry. (2006) 59:395400. doi: 10.1016/j.biopsych.2005.07.036
62. Levinson AJ, Fitzgerald PB, Favalli G, Blumberger DM, Daigle M, Daskalakis ZJ. Evidence of cortical inhibitory deficits in major depressive disorder. Biol Psychiatry. (2010) 67:458-64. doi: 10.1016/j.biopsych.2009.09.025

63. Croarkin PE, Nakonezny PA, Husain MM, Melton T, Buyukdura JS, Kennard $\mathrm{BD}$, et al. Evidence for increased glutamatergic cortical facilitation in children and adolescents with major depressive disorder. JAMA Psychiatry. (2013) 70:291-9. doi: 10.1001/2013.jamapsychiatry.24

64. Luborzewski A, Schubert F, Seifert F, Danker-Hopfe H, Brakemeier EL, Schlattmann P, et al. Metabolic alterations in the dorsolateral prefrontal cortex after treatment with high-frequency repetitive transcranial magnetic stimulation in patients with unipolar major depression. J Psychiatr Res. (2007) 41:606-15. doi: 10.1016/j.jpsychires.2006.02.003

65. Yang XR, Kirton A, Wilkes TC, Pradhan S, Liu I, Jaworska N, et al. Glutamate alterations associated with transcranial magnetic stimulation in youth depression: a case series. J ECT. (2014) 30:242-7. doi: 10.1097/YCT.0000000000000094

66. Peng Z, Zhou C, Xue S, Bai J, Yu S, Li X, et al. Mechanism of repetitive transcranial magnetic stimulation for depression. Shanghai Arch Psychiatry. (2018) 30:84-92.

67. Levitt JG, Kalender G, O'Neill J, Diaz JP, Cook IA, Ginder N, et al. Dorsolateral prefrontal gamma-aminobutyric acid in patients with treatment-resistant depression after transcranial magnetic stimulation measured with magnetic resonance spectroscopy. J Psychiatry Neurosci. (2019) 44:386-94. doi: 10.1503/jpn.180230

68. Davidson RJ, Pizzagalli D, Nitschke JB, Putnam K. Depression: perspectives from affective neuroscience. Annu Rev Psychol. (2002) 53:545-74. doi: 10.1146/annurev.psych.53.100901.135148

69. Drevets WC. Functional neuroimaging studies of depression: the anatomy of melancholia. Annu Rev Med. (1998) 49:34161. doi: 10.1146/annurev.med.49.1.341

70. Anand A, Shekhar A. Brain imaging studies in mood and anxiety disorders: special emphasis on the amygdala. Ann N Y Acad Sci. (2003) 985:37088. doi: 10.1111/j.1749-6632.2003.tb07095.x

71. Drevets WC. Neuroimaging and neuropathological studies of depression: implications for the cognitive-emotional features of mood disorders. Curr Opin Neurobiol. (2001) 11:240-9. doi: 10.1016/S0959-4388(00)00203-8

72. Koenigs M, Grafman J. The functional neuroanatomy of depression: distinct roles for ventromedial and dorsolateral prefrontal cortex. Behav Brain Res. (2009) 201:239-43. doi: 10.1016/j.bbr.2009.03.004

73. Brunoni AR, Vanderhasselt MA. Working memory improvement with non-invasive brain stimulation of the dorsolateral prefrontal cortex: a systematic review and meta-analysis. Brain Cogn. (2014) 86:1-9. doi: 10.1016/j.bandc.2014.01.008

74. Fitzgerald PB, Oxley TJ, Laird AR, Kulkarni J, Egan GF, Daskalakis ZJ. An analysis of functional neuroimaging studies of dorsolateral prefrontal cortical activity in depression. Psychiatry Res. (2006) 148:3345. doi: 10.1016/j.pscychresns.2006.04.006

75. Grimm S, Beck J, Schuepbach D, Hell D, Boesiger P, Bermpohl F, et al. Imbalance between left and right dorsolateral prefrontal cortex in major depression is linked to negative emotional judgment: an fMRI study in severe major depressive disorder. Biol Psychiatry. (2008) 63:36976. doi: 10.1016/j.biopsych.2007.05.033

76. Oh DH, Oh D, Son H, Webster MJ, Weickert CS, Kim SH. An association between the reduced levels of SLC1A2 and GAD1 in the dorsolateral prefrontal cortex in major depressive disorder: possible involvement of an attenuated RAF/MEK/ERK signaling pathway. J Neural Transm. (2014) 121:783-92. doi: 10.1007/s00702-014-1189-z

77. Michael N, Gosling M, Reutemann M, Kersting A, Heindel W, Arolt V, et al. Metabolic changes after repetitive transcranial magnetic stimulation (rTMS) of the left prefrontal cortex: a sham-controlled proton magnetic resonance spectroscopy (1H MRS) study of healthy brain. Eur J Neurosci. (2003) 17:2462-8. doi: 10.1046/j.1460-9568.2003.02683.x

78. Michael N, Erfurth A, Ohrmann P, Arolt V, Heindel W, Pfleiderer B. Metabolic changes within the left dorsolateral prefrontal cortex occurring with electroconvulsive therapy in patients with treatment resistant unipolar depression. Psychol Med. (2003) 33:1277-84. doi: 10.1017/S0033291703007931 
79. Drevets WC, Price JL, Simpson JR, Jr., Todd RD, Reich T, et al. Subgenual prefrontal cortex abnormalities in mood disorders. Nature. (1997) 386:8247. doi: $10.1038 / 386824 \mathrm{a} 0$

80. Alexopoulos GS, Hoptman MJ, Kanellopoulos D, Murphy CF, Lim KO, Gunning FM. Functional connectivity in the cognitive control network and the default mode network in late-life depression. J Affect Disord. (2012) 139:56-65. doi: 10.1016/j.jad.2011.12.002

81. Drevets WC, Price JL, Furey ML. Brain structural and functional abnormalities in mood disorders: implications for neurocircuitry models of depression. Brain Struct Funct. (2008) 213:93-118. doi: 10.1007/s00429-008-0189-x

82. Cummings JL. Frontal-subcortical circuits and human behavior. Arch Neurol. (1993) 50:873-80. doi: 10.1001/archneur.1993.00540080076020

83. Mayberg HS. Modulating dysfunctional limbic-cortical circuits in depression: towards development of brain-based algorithms for diagnosis and optimised treatment. Br Med Bull. (2003) 65:193-207. doi: 10.1093/bmb/65.1.193

84. Grimm S, Luborzewski A, Schubert F, Merkl A, Kronenberg G, Colla M, et al. Region-specific glutamate changes in patients with unipolar depression. J Psychiatr Res. (2012) 46:1059-65. doi: 10.1016/j.jpsychires.2012.04.018

85. American Psychatric Association. Diagnostic and Statistical Manual of Mental Disorders. 4th Text Rev ed. Washington, DC: American Psychatric Association (2000).

86. Downar J, Geraci J, Salomons TV, Dunlop K, Wheeler S, McAndrews $\mathrm{MP}$, et al. Anhedonia and reward-circuit connectivity distinguish nonresponders from responders to dorsomedial prefrontal repetitive transcranial magnetic stimulation in major depression. Biol Psychiatry. (2014) 76:176-85. doi: 10.1016/j.biopsych.2013.10.026

87. Salomons TV, Dunlop K, Kennedy SH, Flint A, Geraci J, Giacobbe $\mathrm{P}$, et al. Resting-state cortico-thalamic-striatal connectivity predicts response to dorsomedial prefrontal rTMS in major depressive disorder. Neuropsychopharmacology. (2014) 39:488-98. doi: 10.1038/npp.2013.222

88. Fitzgerald PB, Huntsman S, Gunewardene R, Kulkarni J, Daskalakis ZJ. A randomized trial of low-frequency right-prefrontal-cortex transcranial magnetic stimulation as augmentation in treatmentresistant major depression. Int J Neuropsychopharmacol. (2006) 9:655-66. doi: 10.1017/S1461145706007176

89. Januel D, Dumortier G, Verdon CM, Stamatiadis L, Saba G, Cabaret W, et al. A double-blind sham controlled study of right prefrontal repetitive transcranial magnetic stimulation (rTMS): therapeutic and cognitive effect in medication free unipolar depression during 4 weeks. Prog Neuropsychopharmacol Biol Psychiatry. (2006) 30:126-30. doi: 10.1016/j.pnpbp.2005.08.016

90. Gruetter R. Automatic, localized in vivo adjustment of all first- and second-order shim coils. Magn Reson Med. (1993) 29:804-11. doi: 10.1002/mrm.1910290613

91. Naressi A, Couturier C, Devos JM, Janssen M, Mangeat C, de Beer R, et al. Java-based graphical user interface for the MRUI quantitation package. Magma. (2001) 12:141-52. doi: 10.1007/BF02668096

92. Bhattacharyya PK, Phillips MD, Stone LA, Bermel RA, Lowe MJ. Sensorimotor cortex gamma-aminobutyric acid concentration correlates with impaired performance in patients with MS. AJNR Am J Neuroradiol. (2013) 34:1733-9. doi: 10.3174/ajnr.A3483

93. Pijnappel WWF, Van den Boogaart A, De Beer R, Van Ormondt D. SVDbased quantification of magnetic resonance signals. J Magn Reson. (1992) 97:122-4. doi: 10.1016/0022-2364(92)90241-X

94. Vanhamme L, van den Boogaart A, Van Huffel S. Improved method for accurate and efficient quantification of MRS data with use of prior knowledge. J Magn Reson. (1997) 129:35-43. doi: 10.1006/jmre.1997.1244

95. Chang L, Jiang CS, Ernst T. Effects of age and sex on brain glutamate and other metabolites. Magn Reson Imaging. (2009) 27:142-5. doi: 10.1016/j.mri.2008.06.002

96. Salvadore G, van der Veen JW, Zhang Y, Marenco S, MachadoVieira R, Baumann $\mathrm{J}$, et al. An investigation of amino-acid neurotransmitters as potential predictors of clinical improvement to ketamine in depression. Int J Neuropsychopharmacol. (2012) 15:1063-72. doi: 10.1017/S1461145711001593

97. Mescher M, Merkle H, Kirsch J, Garwood M, Gruetter R. Simultaneous in vivo spectral editing and water suppression. NMR Biomed. (1998) 11:26672. doi: 10.1002/(SICI)1099-1492(199810)11:6<266::AID-NBM530>3.0. $\mathrm{CO} ; 2-\mathrm{J}$

98. Terpstra M, Ugurbil K, Gruetter R. Direct in vivo measurement of human cerebral GABA concentration using MEGA-editing at 7 Tesla. Magn Reson Med. (2002) 47:1009-12. doi: 10.1002/mrm.10146

99. Milak MS, Proper CJ, Mulhern ST, Parter AL, Kegeles LS, Ogden RT, et al. A pilot in vivo proton magnetic resonance spectroscopy study of amino acid neurotransmitter response to ketamine treatment of major depressive disorder. Mol Psychiatry. (2016) 21:320-7. doi: 10.1038/mp.2015.83

100. Cleve M, Gussew A, Reichenbach JR. In vivo detection of acute paininduced changes of GABA+ and Glx in the human brain by using functional 1H MEGA-PRESS MR spectroscopy. Neuroimage. (2015) 105:6775. doi: 10.1016/j.neuroimage.2014.10.042

101. de la Fuente-Sandoval C, Reyes-Madrigal F, Mao X, Leon-Ortiz P, Rodriguez-Mayoral O, Solis-Vivanco R, et al. Cortico-striatal GABAergic and glutamatergic dysregulations in subjects at ultra-high risk for psychosis investigated with proton magnetic resonance spectroscopy. Int $J$ Neuropsychopharmacol. (2015) 19:pyv105. doi: 10.1093/ijnp/pyv105

102. Yang XR, Langevin LM, Jaworska N, Kirton A, Lebel RM, Harris AD, et al. Proton spectroscopy study of the dorsolateral prefrontal cortex in youth with familial depression. Psychiatry Clin Neurosci. (2016) 70:26977. doi: $10.1111 / \mathrm{pcn} .12392$

103. Mikkelsen M, Barker PB, Bhattacharyya PK, Brix MK, Buur PF, Cecil KM, et al. Big GABA: edited MR spectroscopy at 24 research sites. Neuroimage. (2017) 159:32-45. doi: 10.1016/j.neuroimage.2017.07.021

104. Ramadan S, Lin A, Stanwell P. Glutamate and glutamine: a review of in vivo MRS in the human brain. NMR Biomed. (2013) 26:163046. doi: 10.1002/nbm.3045

105. Goto N, Yoshimura R, Kakeda S, Nishimura J, Moriya J, Hayashi K, et al. Sixmonth treatment with atypical antipsychotic drugs decreased frontal-lobe levels of glutamate plus glutamine in early-stage first-episode schizophrenia. Neuropsychiatr Dis Treat. (2012) 8:119-22. doi: 10.2147/NDT.S25582

106. Bhattacharyya PK. Macromolecule contamination in GABA editing using MEGA-PRESS should be properly accounted for. Neuroimage. (2014) 84:1111-2. doi: 10.1016/j.neuroimage.2013.08.050

107. Ahdab R, Ayache SS, Brugieres P, Goujon C, Lefaucheur JP. Comparison of "standard" and "navigated" procedures of TMS coil positioning over motor, premotor and prefrontal targets in patients with chronic pain and depression. Neurophysiol Clin. (2010) 40:27-36. doi: 10.1016/j.neucli.2010.01.001

Conflict of Interest: The authors declare that the research was conducted in the absence of any commercial or financial relationships that could be construed as a potential conflict of interest.

Publisher's Note: All claims expressed in this article are solely those of the authors and do not necessarily represent those of their affiliated organizations, or those of the publisher, the editors and the reviewers. Any product that may be evaluated in this article, or claim that may be made by its manufacturer, is not guaranteed or endorsed by the publisher.

Copyright (c) 2021 Bhattacharyya, Anand, Lin and Altinay. This is an open-access article distributed under the terms of the Creative Commons Attribution License (CC BY). The use, distribution or reproduction in other forums is permitted, provided the original author(s) and the copyright owner(s) are credited and that the original publication in this journal is cited, in accordance with accepted academic practice. No use, distribution or reproduction is permitted which does not comply with these terms. 